Journal of Advanced Research in Applied Sciences and Engineering Technology

\title{
A Design of New Product Database System for Supporting Step-Compliant Total Integration Applications
}

\author{
Chen Wong Keong ${ }^{1}$, Yusri Yusof ${ }^{2, *}$ \\ Department of Mechanical Engineering, Polytechnic Mukah, 96400 Mukah, Sarawak, Malaysia \\ 2 Department of Manufacturing and Industry Engineering, Faculty Mechanical \& Manufacturing Engineering, Universiti Tun Hussein Onn Malaysia \\ Parit Raja, Johor, 86400, Malaysia
}

\begin{abstract}
Computer technology has become a very important element in an advanced manufacturing system. The good and systematic data model for the exchange of manufacturing information between different stages of development of product life cycle is paramount to ensure the product is manufactured and delivered to the market successfully. As a result, ISO 10303, an international standard, or well known as Standard for Exchange of Product Model Data (STEP) is not just for providing a neutral data format within the heterogenous CAD systems, its functionality has extended to the whole life cycle of product. STEP Part 21 is the first implementation method from EXPRESS modelling language and implemented successfully as neutral product data to integrate heterogeneous CAD platform. However, this CAD STEP Part21 text file is hardly to be applied in manufacturing processes since it consists of purely geometrical and topological data. In this research, a nonprocedural approach is presented to translate the EXPRESS language model of STEP CAD data into a new product database system model. A new nonprocedural approach of data enrichment and automated machining feature recognition is proposed and implemented on this newly developed product database system and provide a solution for the interrelated tasks of automated machining feature recognition: (1) extraction of geometrical feature from STEP CAD model data of the part (2) formation of part representation suitable for form feature identification (3) matching of form features. This paper proves the validity of this newly developed product database system by translating STEP Part21 file from a commercial CAD system to database system format, data enrichment, performing automated machining feature recognition and lastly generating STEP standard data according to AP224 for supporting STEP based process planning and manufacturing applications.
\end{abstract}

\section{Keywords:}

Feature Extraction, Automated Machining Feature Recognition, Database system

Copyright @ 2020 PENERBIT AKADEMIA BARU - All rights reserved

\section{Introduction}

Industrial manufacturing is one of the most important sources of wealth for a country. The advanced of industrial manufacturing is not only able to provide daily needs for our own society, but exporting the high quality products to all over the world [3]. The advent of computer has change the landscape of our current trend of manufacturing systems significantly. Computer has brought a lot of advantages to the life cycle of product development, from the initial stage of product design, product manufacturing, product maintenance, and also dispose of the end product. Commercialized

\footnotetext{
* Corresponding author.

E-mail address: yusri@uthm.edu.my
}

https://doi.org/10.37934/araset.18.1.113 
software solutions and industrial systems that related to computer aided design, process planning, manufacturing and numerical control are readily available due to today's technological developments $[6,7]$.

CAD/CAM software is used to design and manufacture prototypes, finished products, and production runs [1]. An integrated CAD/CAM system offers one complete solution for design through manufacturing. CAPP is the use of computer technology to aid in the process planning of a part or product, in manufacturing [2]. CAPP is the link between CAD and CAM in that it provides for the planning of the process to be used in producing a designed part. Numerical control (also computer numerical control, and commonly called $\mathrm{CNC}$ ) is the automated control of machining tools (drills, boring tools, lathes) and 3D printers by means of a computer. A CNC machine processes a piece of material (metal, plastic, wood, ceramic, or composite) to meet specifications by following a coded programmed instruction and without a manual operator [8]. In term of total integration of all these software, the integrating Computer-Aided Design (CAD)/Computer-Aided Manufacture (CAM)/Computer-aided process planning (CAPP)/Computer Numerical Control (CNC) can be achieved through data exchange by utilizing STEP and STEP-NC as shown in the Figure 1 [9].

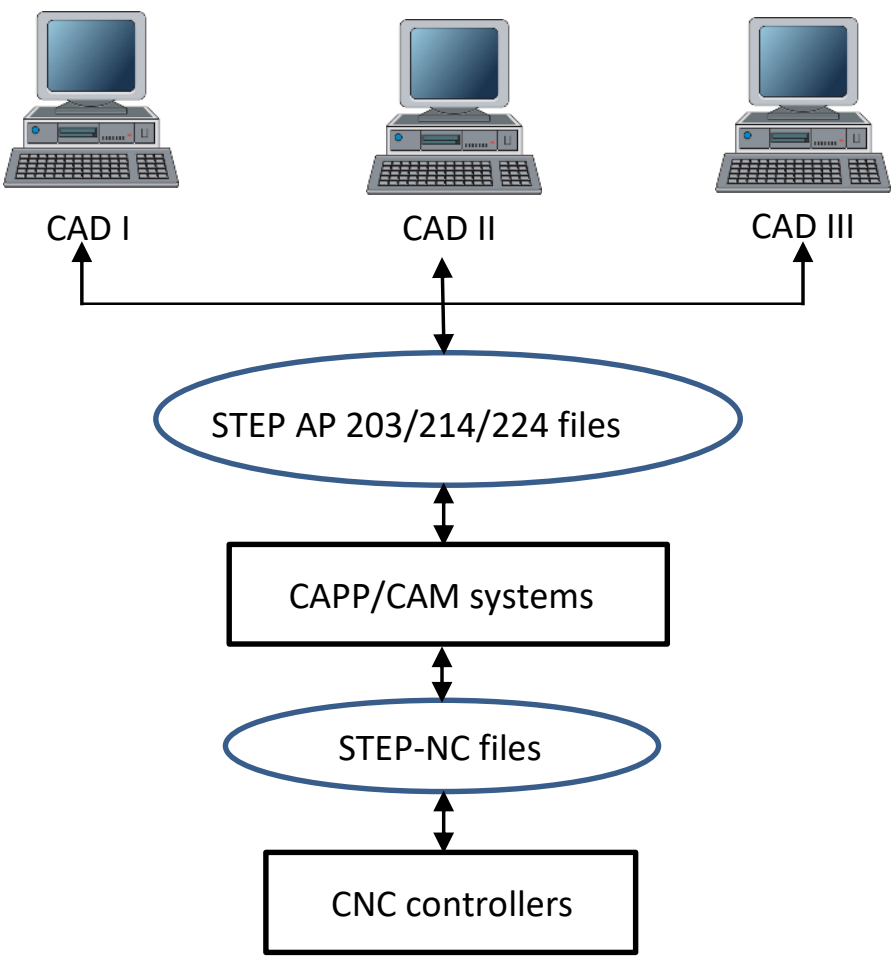

Fig. 1. Striving for CAD/ CAP/ CAM/ CNC integration through STEP and STEP NC

ISO 10303, an international standard, or well known as Standard for Exchange of Product Model Data (STEP) is not just for providing a neutral data format within the heterogenous CAD systems, its functionality has extended to the whole life cycle of product. STEP consists of different application protocols (AP) with different main applications. AP203, AP214, AP219 and AP224 are the examples of the Application Protocols that used to define the industry data for design and manufacturing [10]. In the product life cycle, all of these APs are used to define different data representations at different levels. However, the data scope and context such as geo-dimensionality and tolerance, geometry, raw material data, boundary representation geometry and machining characteristics for all APs are similar among each other [4]. Although many researchers have the efforts on developing APs, but 
harmonizing these APs is still in the process. The development of AP in integrated manufacturing systems is also being studied [11].

Different types of CAD conversions depend on different companies. When product models are accepted in a neutral data exchange format such as the Standard for Exchange product (STEP) data model (ISO 10303-1, 1994) and Early Graphic Exchange Standard (IGES), companies may choose to "Rebuild" or redo features based on accepted data models.

This exercise is called "re-mastering" the model. Re-mastering is needed due to the STEPS and IGES can only reflect the geometric data and pure topology of the model (dummy data model, similar to the model from a historical independent CAD system) except for all other product-related data such as design and tolerance features. Alternatively, a company may simply abandon it as it can be a costly exercise to regain control of the model.

Therefore, the main objective of this research is to develop a new relational database management system with adopting a nonprocedural approach in the effort of harmonizing all these APs, especially between AP203, AP224, and STEP NC in order to support the effort of total integration of CAD/CAM/CAPP/CNC through STEP and STEP NC.

\section{Methodology}

This research framework shows that CAD STEP Part21 file can be generated mostly from any commercialized CAD software. Generally, CAD STEP Part21 is just consist of geometric and topological rich data format, such as vertices, lines and surfaces as shown in Figure 2.

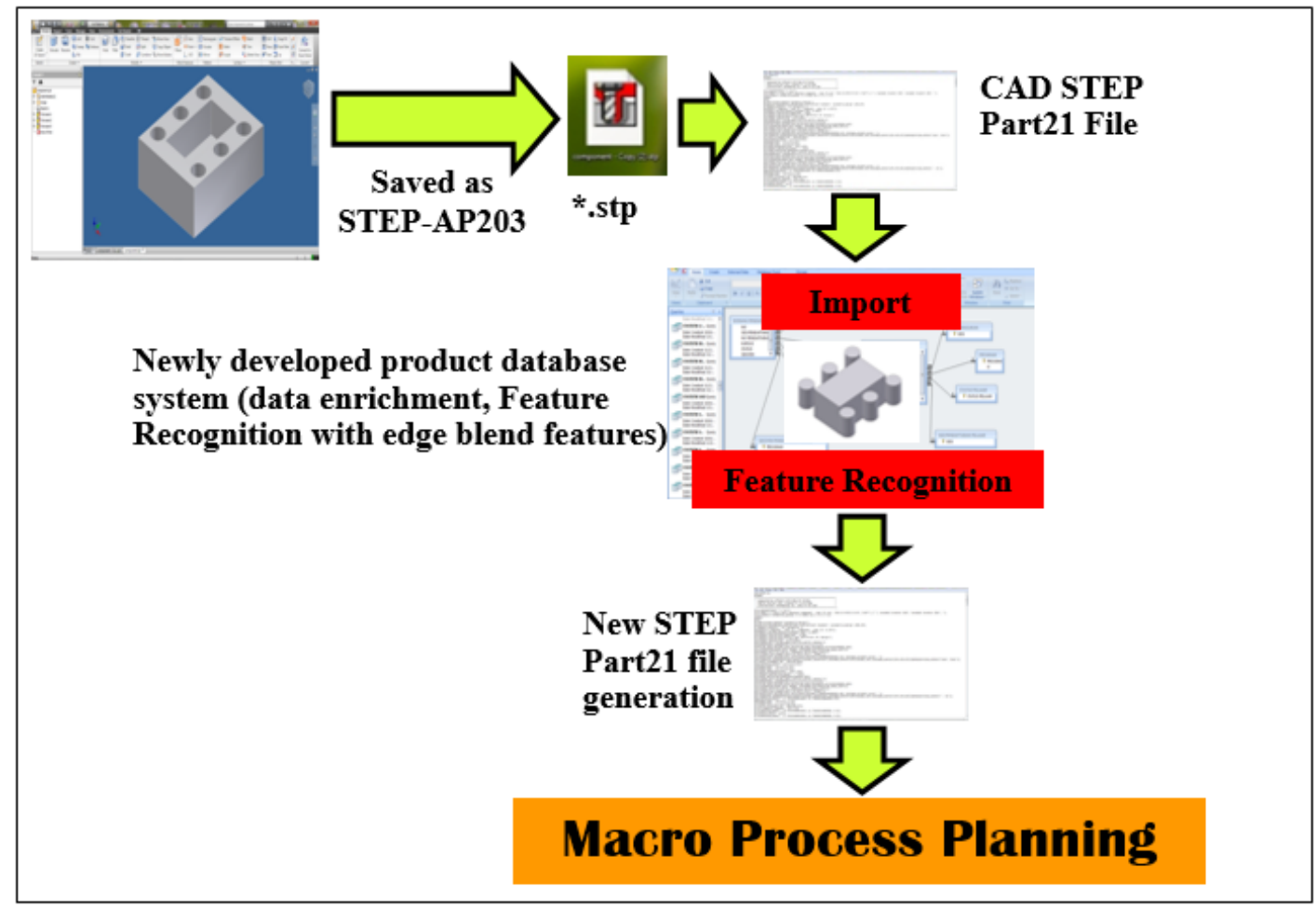

Fig. 2. Project Framework 
This type of data could not be fully utilized to process planning and manufacturing which require more feature based data. As a result, the objectives of this research are to develop a new product database system with nonprocedural approach to extract important information from CAD STEP Part21 file and conducting a series of enrichment process on the data. Then, this product database system could recognize simple and compound machining features automatically from the enriched extracted geometrical and topological data from CAD STEP Part21 file. Lastly, a new STEP Part21 file could be generated according to AP224 format and it is more beneficial for process planning and manufacturing processes within the life cycle of product development based on STEP. In procedural approach, the program code is written as a sequence of instruction by specifying "what to do" and also "how to do" (step by step procedure). However, in nonprocedural approach, program language is written by specifying "what to do" in a higher level functional language. The benefits of nonprocedural language are it is a function-driven language, its semantics are simple, size of the programme is small and easy to manage relatively.

\subsection{Stages of Project Development}

Figure 3 shows stages of research development according to proposed methodology on how to build a new product database system at stage 1, to extract geometric and topological data from CAD STEP Part21 file in stage 2 . Then, the extracted data goes through a series of data enrichment process at stage 3 and the final stage is recognizing machining feature from enriched extracted geometrical and topological data. The processes from stage 2 to stage 5 have been completed within this developed relational database system by using a nonprocedural approach which is provided within the system.

\subsection{Relational Database Management System (RDMS)}

This section discusses about applications of the developed product database system for supporting STEP compliant manufacturing environment. Furthermore, details about the construction of attributed graph and graph-based feature recognition, generation of STEP AP224 data file, and generation of STEP NC data file have been explained in this section. In this research, a new relational product database management system has been developed for establishing a system for integrating and harmonizing between STEP APs, especially for AP203, AP224 and STEP NC (ISO 14649/ AP 238). This relational product database management system consists of 4 modules, (1) geometric and topological data extraction (2) CAD STEP data enrichment (3) automatic machining feature recognition, (4) generation of a new STEP Part21 file according to AP224 and STEP NC as shown in Figure 4. Based on this proposed system, module 1 and module 2 have been discussed and accomplished in section 3, where a database system used for CAD STEP Part21 data extraction and enrichment have been created successfully. So, the main concentration of this system fall on (i) suggesting a novel graph-based method for machining feature recognition (ii) generating a new STEP Part21 file according to AP224 and STEP NC. 


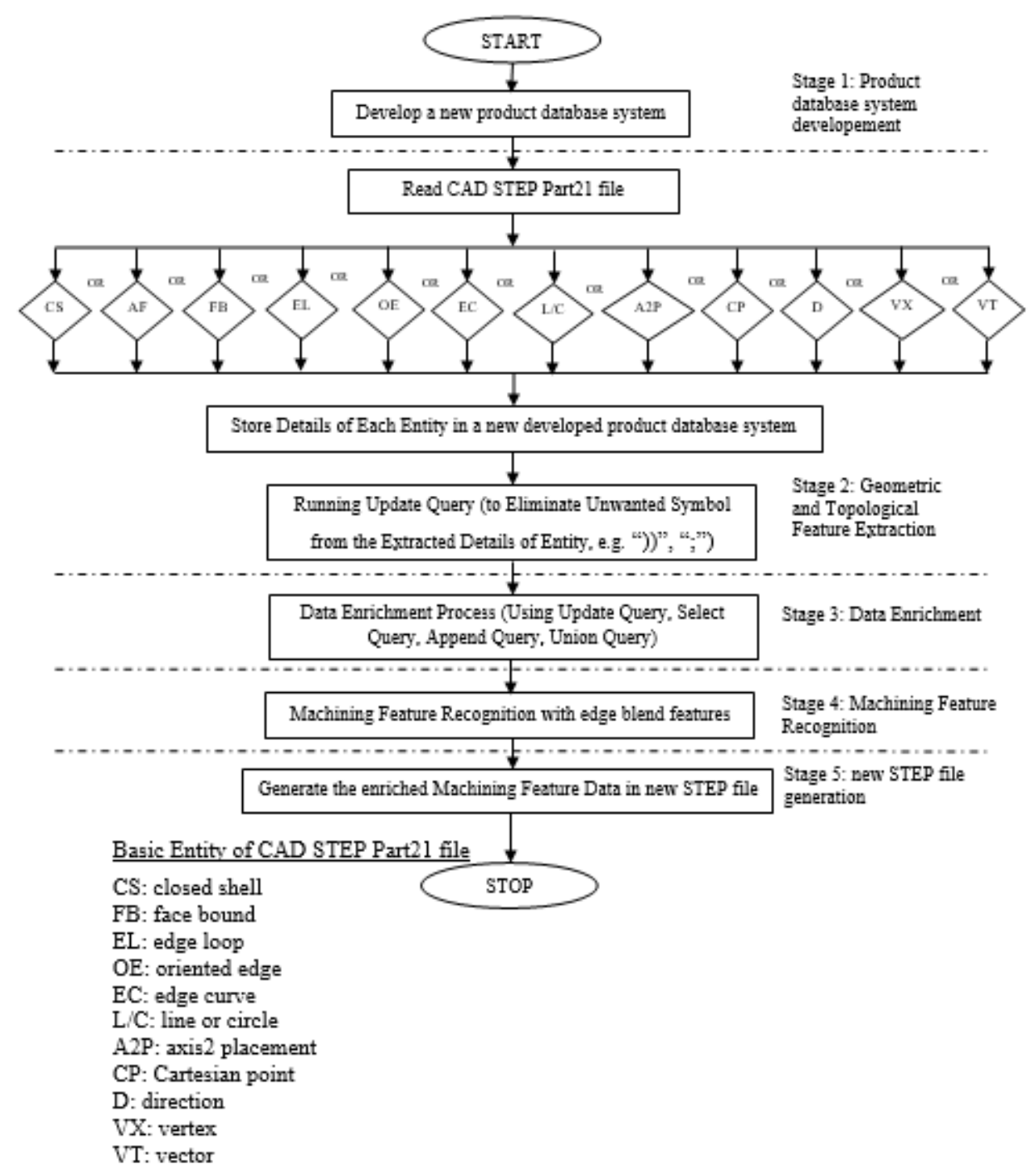

Fig. 3. Five main stages of product database system development

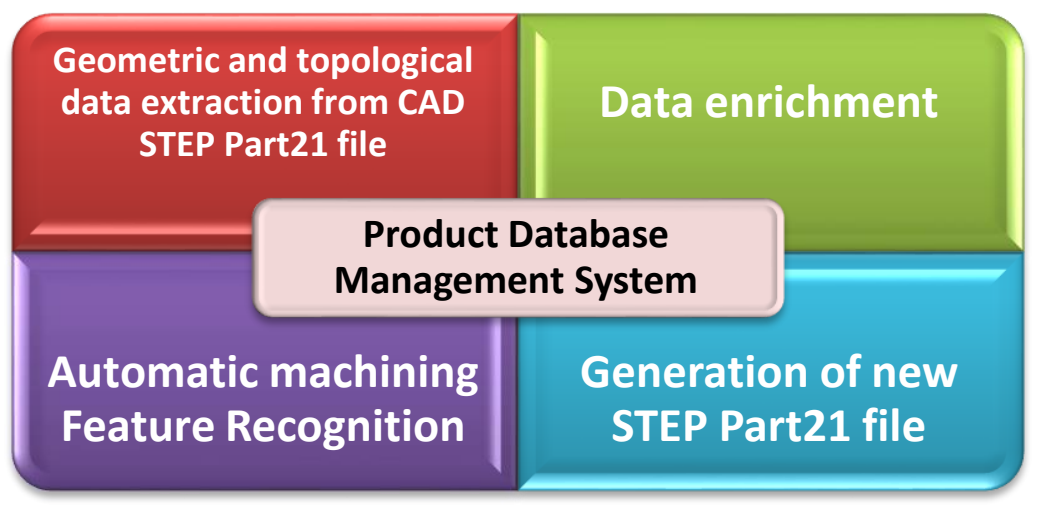

Fig. 4. A proposed framework of relational product database management system for supporting STEP compliant manufacturing environment 


\subsection{Implementation}

The part design is first created by using any commercial computer aided design software, e.g. Autodesk Inventor. The design file is then saved as a neutral data format as STEP AP203 drawing file, which consist of geometric and topological data. The data format is generated according to the ISO 10303-21 standard, or generally known as part 21 file. This type of data is very long and hardly to read by human or computer program. Most programming languages, such as FORTAN, $\mathrm{C}$ and $\mathrm{C}++$, actually read line by line, starting the first to the following line. Due to the nature of data mapping in STEP, backward line reading is required. However, backward reading cannot be carried out by most programming languages unless the reading process is terminated and a new reading process is carried out. Hence, backward reading which results from the characteristics of STEP file is not the solution in writing an efficient source code. The suggestion for the solution of this problem is developing a relational database management system equipped with nonprocedural approach (query) for managing all the geometric and topological data by extracting all of the data and storing the data by types of entities accordingly in different tables. This STEP (CAD) file has been imported to a developed relational database management system (RDBMS). This relational database management system has been developed by using a PC-based RDMS software, Microsoft Access, in Window.

\subsection{Geometric and topological data extraction and machining features recognition from STEP (CAD)} file

The extracted data has been analyzed and regenerated with nonprocedural approach (query) to be more useful data as shown in the Figure 5 and Figure 6 for subsequent automatic machining feature recognition process.

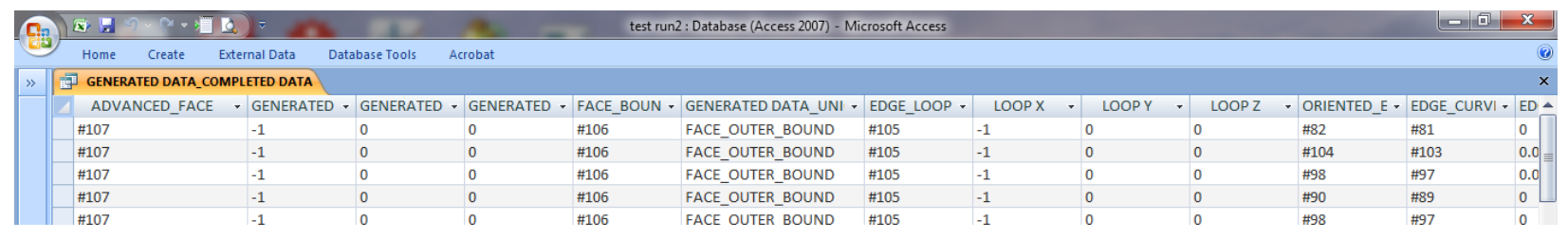

Fig. 5. Some of generated important attributes (direction, types of face bound, related edge curve, related oriented edge) for edge loop of part design

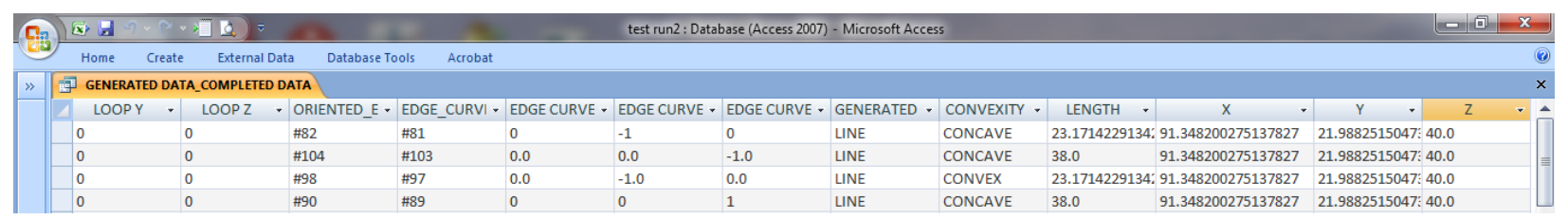

Fig. 6. Some of generated important attributes (types of edge curve, concavity/convexity of edge curve, length and direction of related edge curve) for edge loop of part design 


\subsection{Automatic 2.5D Machining Feature Recognition (A novel graph-based method)}

When dealing with a boundary representation of a design component, instead of faces, edge loop has been proposed as nodes of the graph and edge loops relationships form the arc/links of the graph which is known as attributed edge loop adjacency graph (AELAG). Some of important attributes of edge loop are generated, such as direction of edge loop, collection of edge curves, length of edge curves, concavity/convexity of edge curve, outer bound or inner bound of loop curve from the extracted geometric and topological CAD data. Figure 7 shows a new design of attributed edge loop adjacency graph for pocket recognition and Figure 8 shows the AELAG mapping for recognizing a pocket in RDMS tables format.
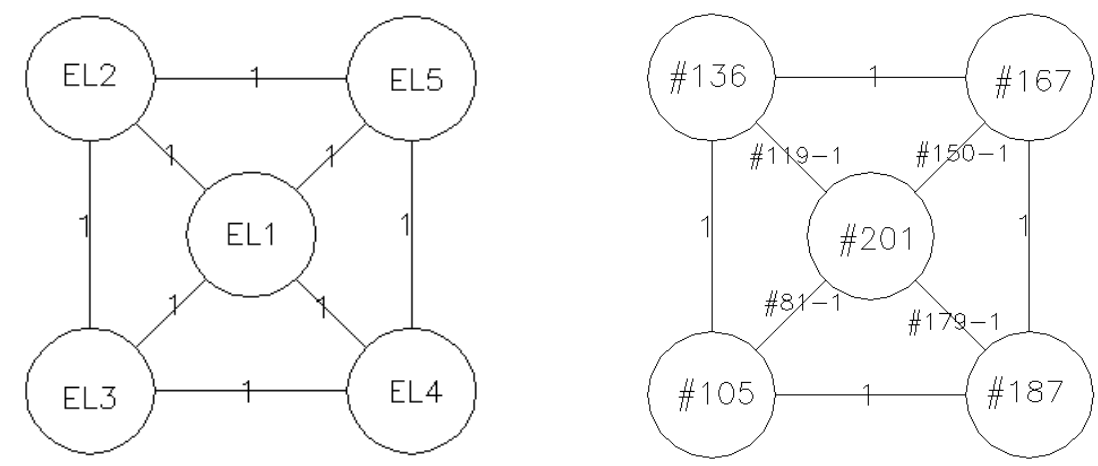

concave: 1 ; convex: 0

Fig. 7. A new design of attributed edge loop adjacency graph (AELAG) for pocket recognition

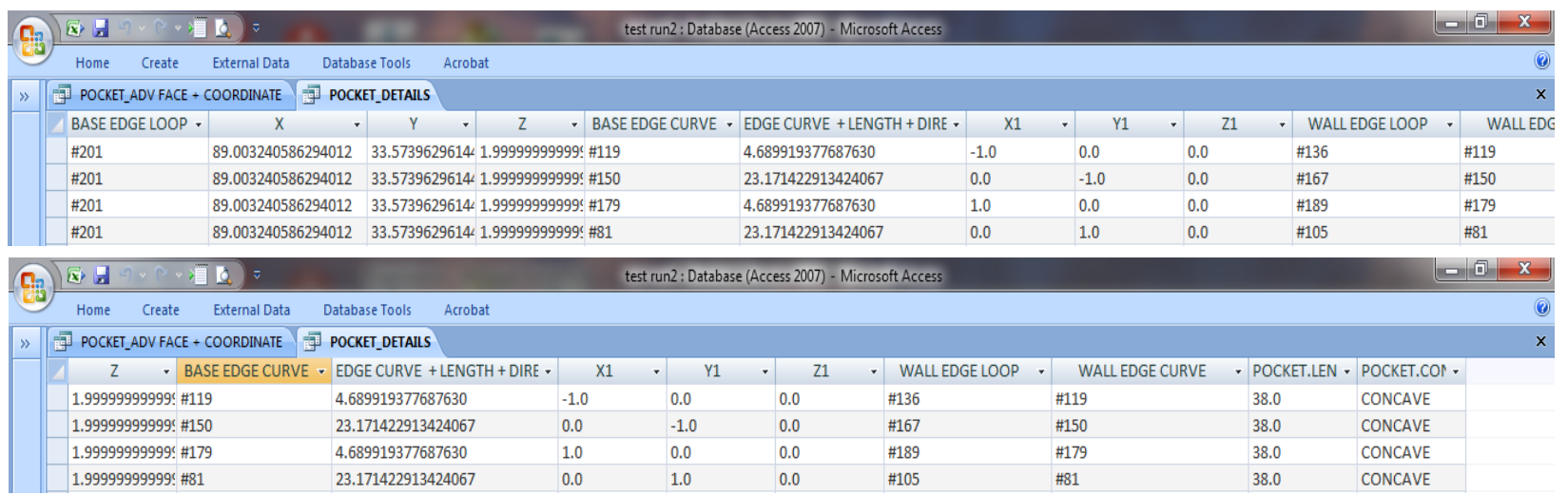

Fig. 8. AELAG mapping for recognizing a pocket in RDMS tables format

\subsection{Generation of STEP AP224 data file}

From recognized machining features with important parameters, such as pocket, the database system is going to generate a more standardized machining feature data, in ISO 10303-224 format and according to the ISO 10303-21 standard. The generated format is shown in Figure 9. 


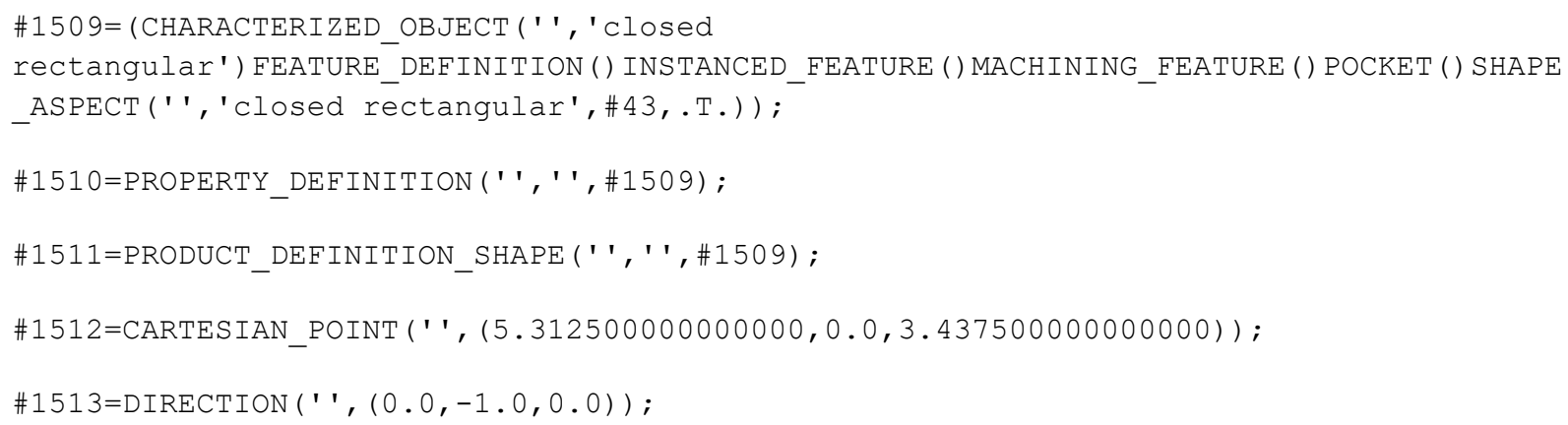

Fig. 9. Machining feature data generated in AP224 format

\subsection{Generation of STEP NC data file}

STEP NC is the extension of using STEP throughout product lifecycle development, especially concentrating on developing a seamless data exchange in the process of machining a recognized machining feature. The part program is described in a Physical File Format according to ISO 1030321. The first section of the part program is the header section marked by the keyword "HEADER". In this header, some general information and comments concerning the part program are given, such as filename, author, date, organization, etc as shown in Figure 10.

Manufacturing feature data typically originate from CAM. ISO 14649 defines manufacturing features that differ from, but are harmonized with, ISO 10303-224. Manufacturing process data also originated form CAM, and defines the technological parameters to be used during the cutting process such as tool feed and spindle speed, and description of the tooling required for each of the machining operations. Manufacturing process data also includes the definitions of Workingsteps, one for each association of feature, of associated tool and its technological parameter, and the sequence of these Workingsteps.

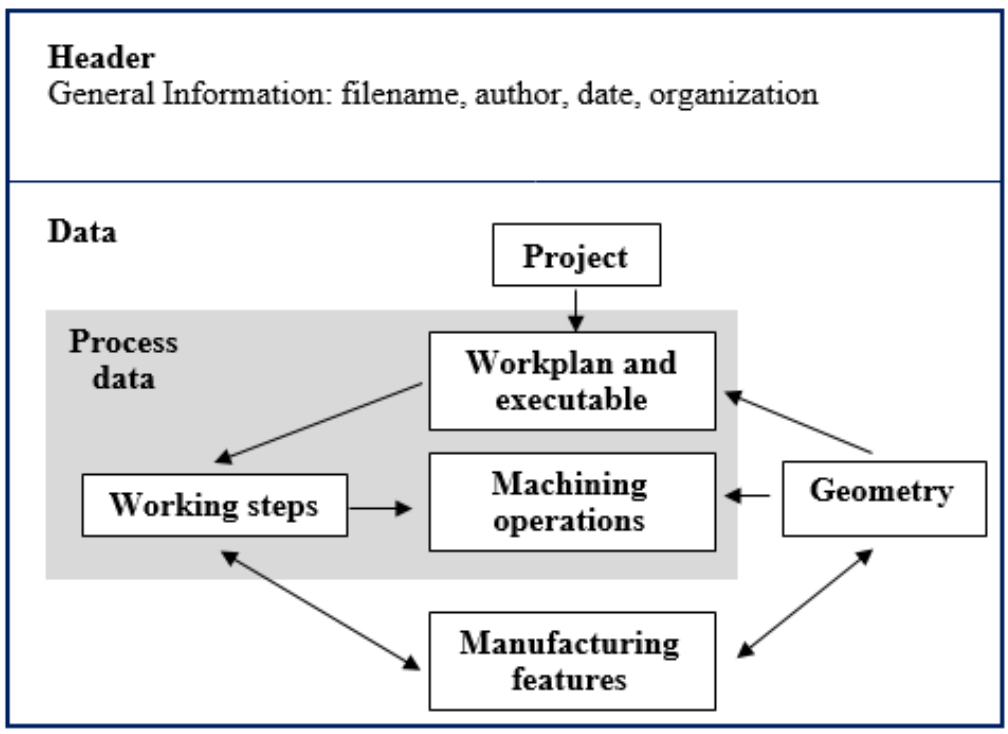

Fig. 10. Data Structure of ISO 14649 [5] 


\section{Result and Discussion}

This section shows the newly developed product database system, named as Automatic Machining Feature Recognition System with Edge Blend Feature, by using window Microsoft Access Relational Database program as shown in Figure 11. A step by step implementation of this system is explained as below.

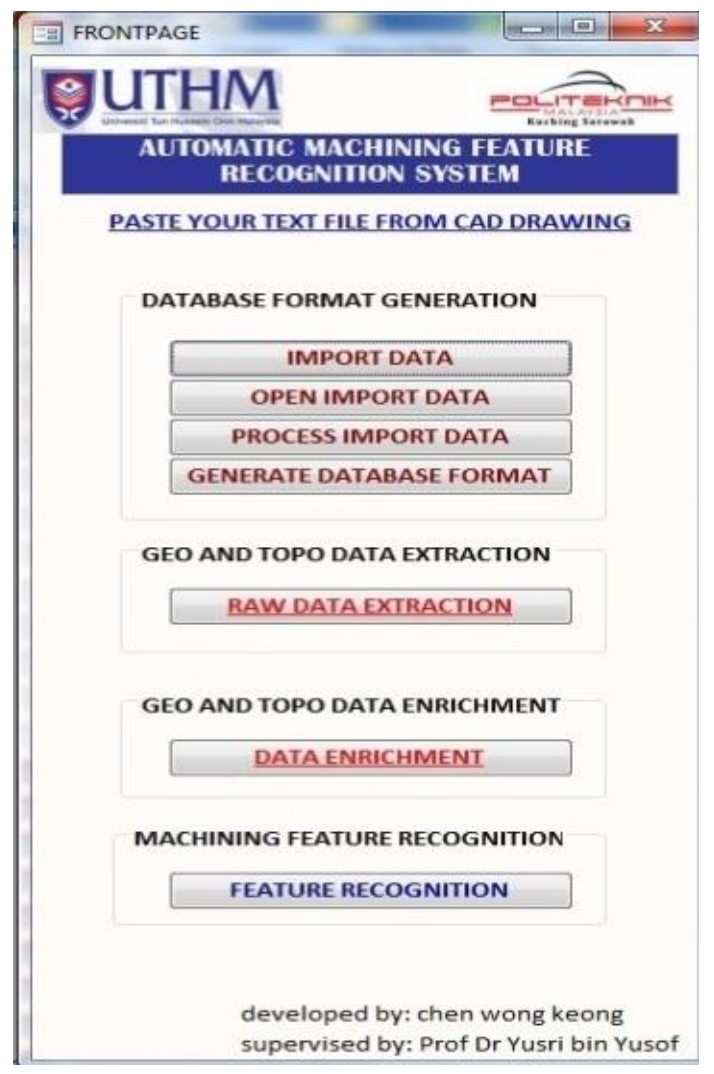

Fig. 11. The interface of the product database system

Implementation procedures are shown as follow: -

Step 1: Design a part using CAD (Autodesk Inventor) and save the file as STEP file ( ${ }^{*}$.stp) as shown in Figure 12.

Step 2: Open the STEP file with Notepad and save as text file (*.txt) as shown in Figure 13.

Step 3: Import the data into the created product database system as shown in Figure 14.

Step 4: Separate the geometry and topological data from STEP file in the following manner

i. Read each line of character from the import STEP file

ii. Extract each line of geometry and topological data entity and then save them into different types of entity tables (e.g. shell table, advanced-face table, edge loop table, face table, edge table, point table and so on)

Step 5: Filter and store all the parameter of the extracted geometry and topological data entity into a sensible data with using the Microsoft Access queries.

Step 6: Display all extracted and enriched product database format in a compact view as shown in Figure 15 and Figure 16.

Step 7: Machining feature recognition as shown in Figure 17 and Figure 18.

Step 8: New Part21 text file generated according to AP224 as shown in Figure 19. 

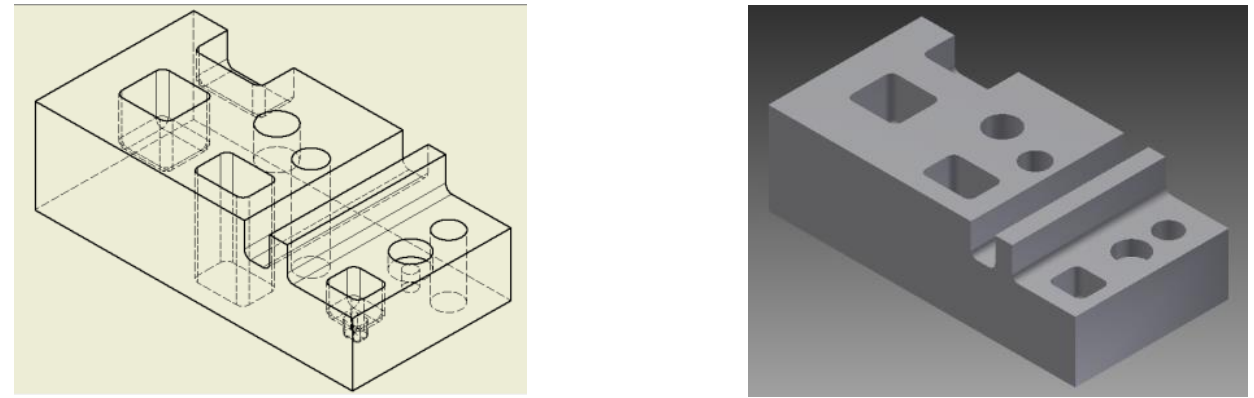

Fig. 12. Part Design created on Autodesk Inventor

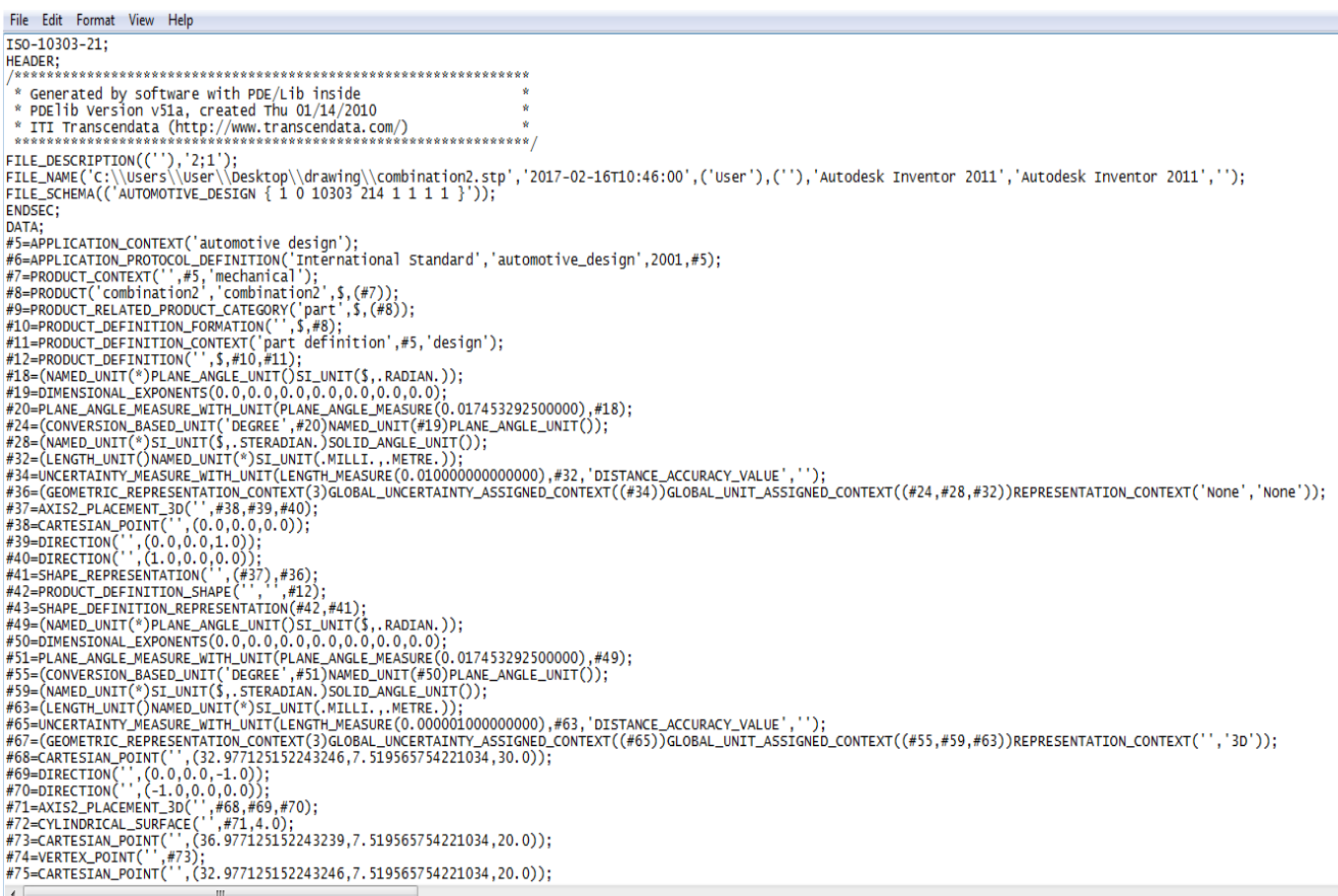

Fig. 13. Text file Part 21 which consists of 2692 lines as internal data structure of a design part

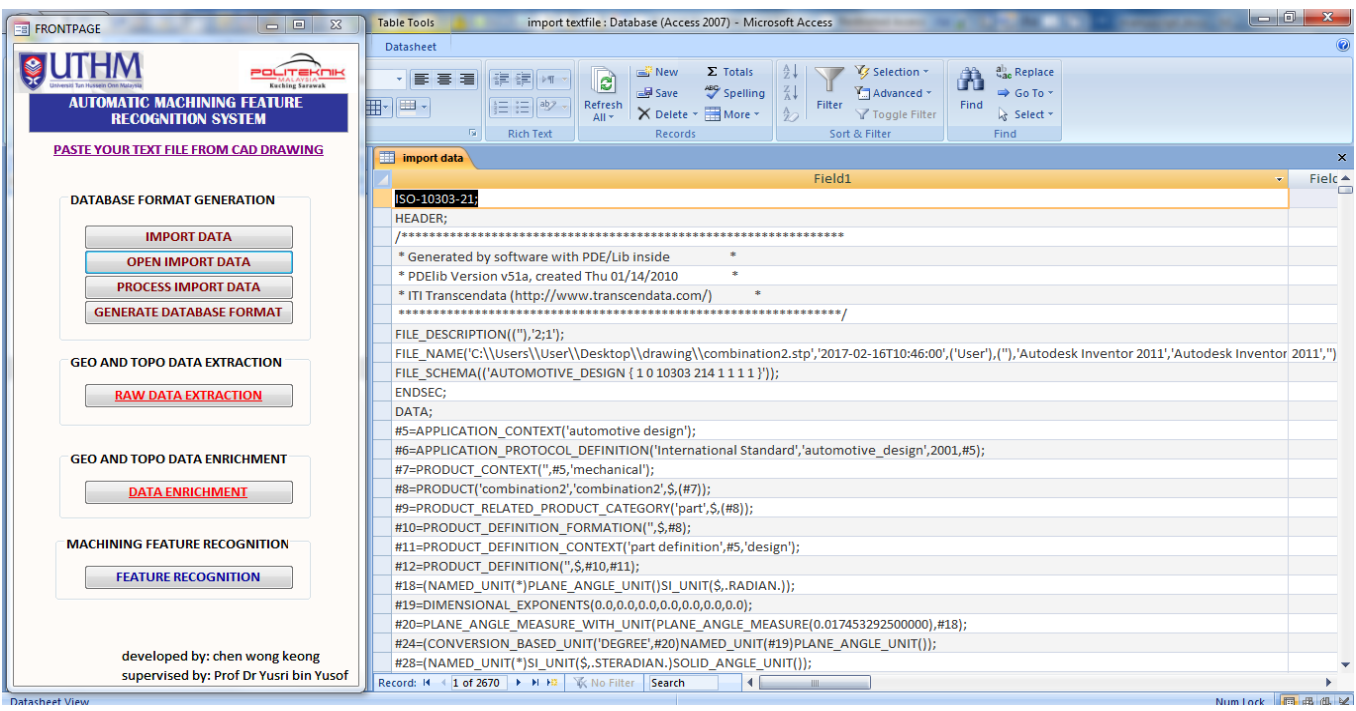

Fig. 14. CAD STEP Part21 file imported to database system 


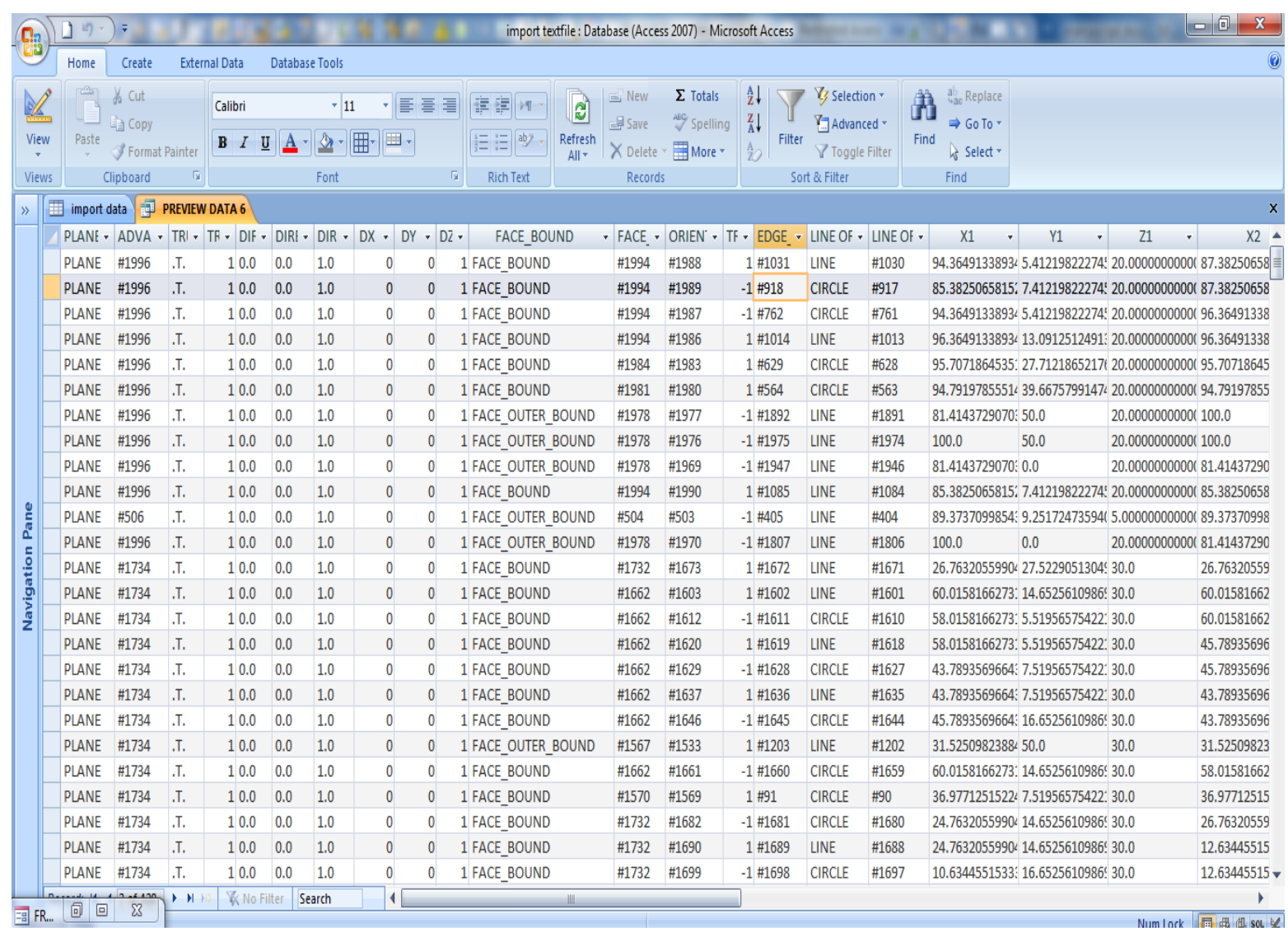

Fig. 15. Geometrical and topological data extraction from CAD STEP Part21 file

The geometry and topological data (in the form of text file) is separated from the CAD STEP Part21 text file by using the query language (query by example method). After completion of the process above, the database has been filled with geometry and topological data and this information will be useful for subsequent process as shown in figure 16,17, 18 and 19.

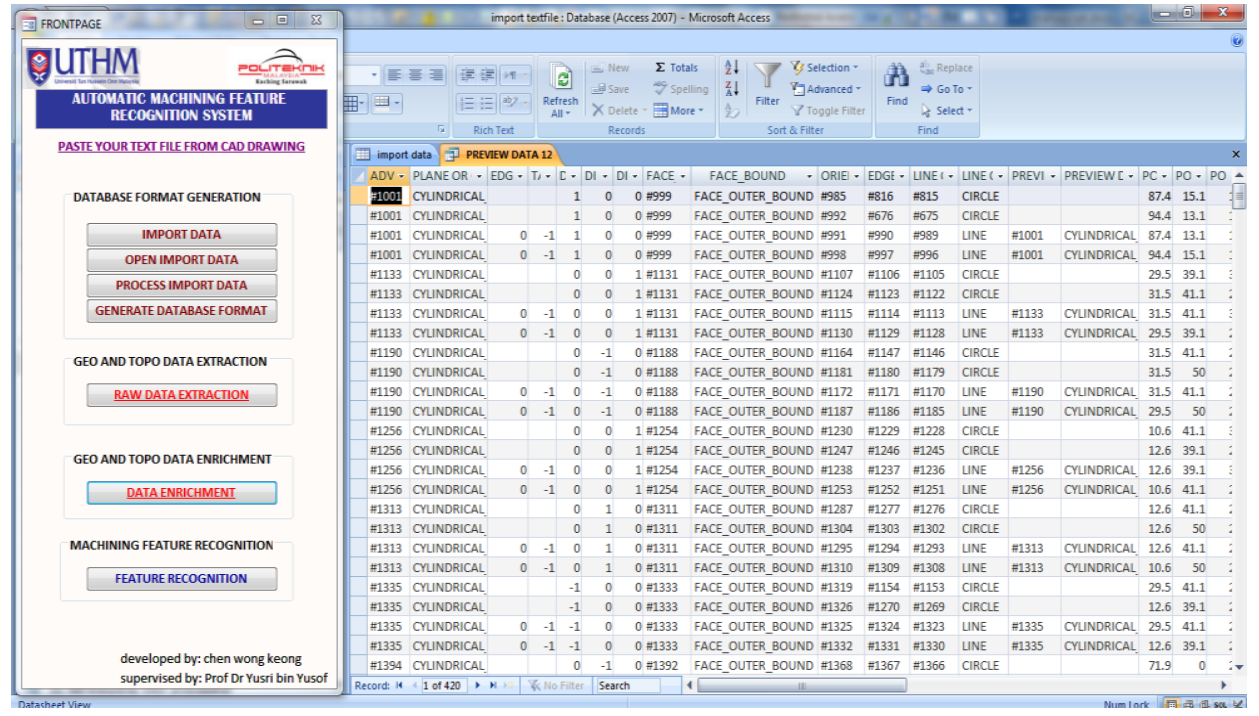

Fig. 16. Extracted and enriched product database format in a compact view which consist of only 420 lines compare with text file Part21 with 2692 lines for describing a design component 


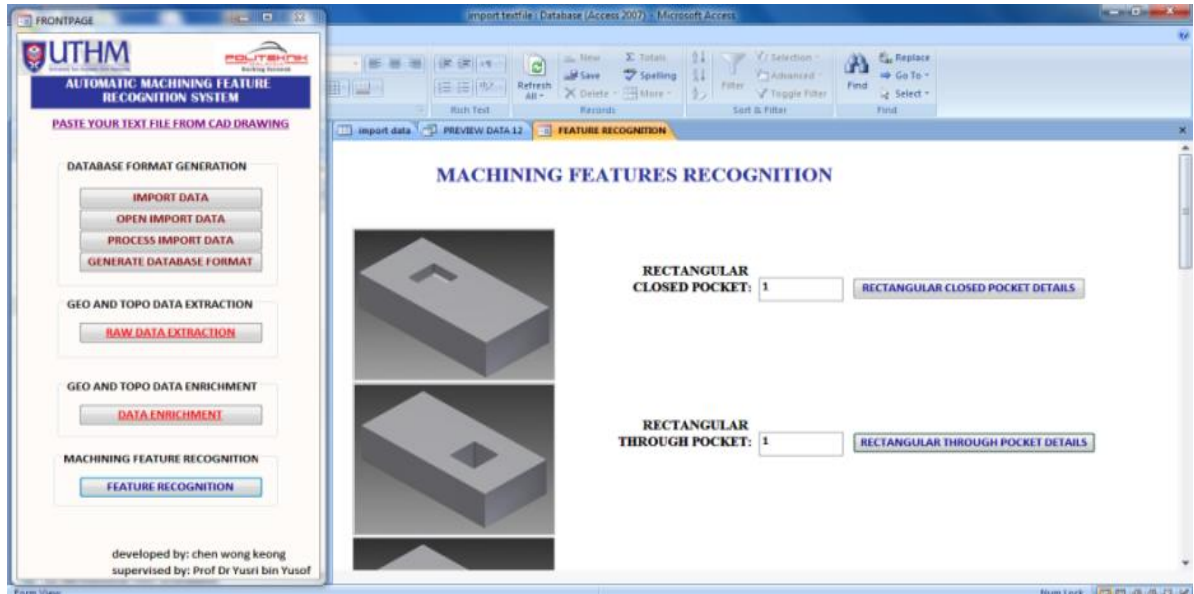

Fig. 17. Recognition of all the machining features

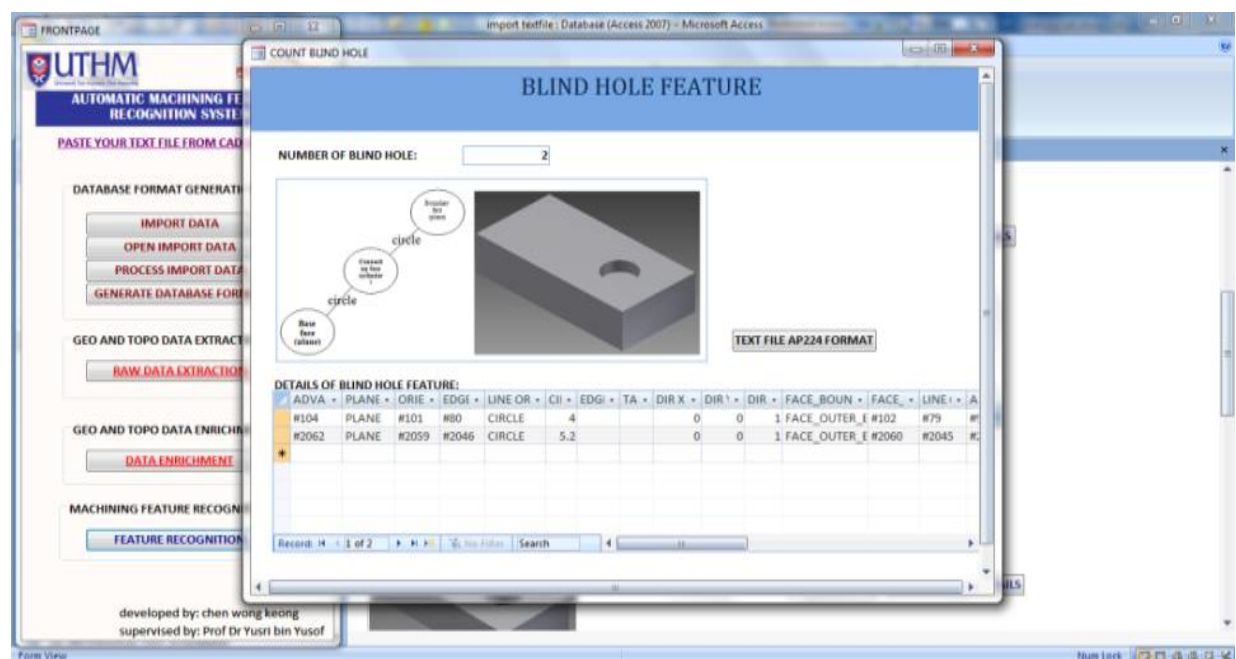

Fig. 18. Details of recognized machining feature (e.g. hole feature)

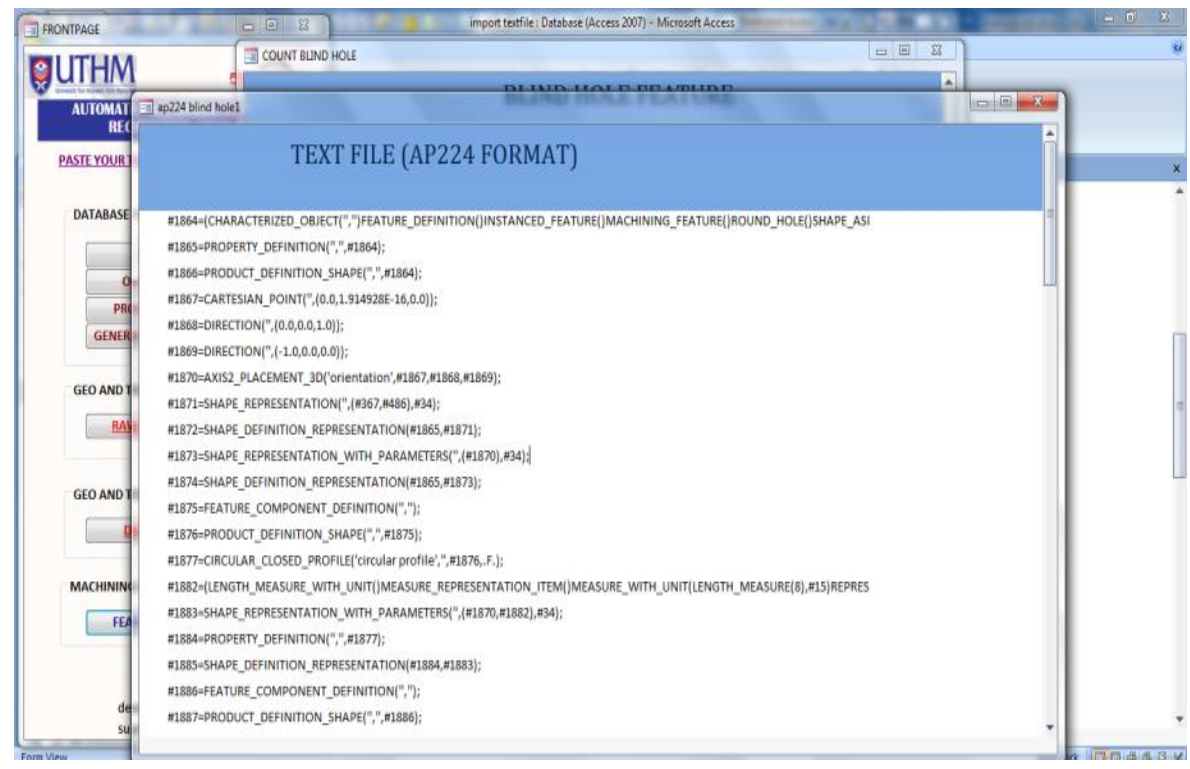

Fig. 19. New Part21 text file generated according to AP224 (e.g. hole feature) 


\section{Conclusion}

In conclusion, this study shows an effort in harmonizing between different STEP APs (AP203, AP224, AP238/ISO 14649) through developing a relational database management system with adopting a nonprocedural approach. A PC-based RDMS software, Microsoft Access, has been introduced for supporting an integrated CAD/ CAPP/ CAM/ CNC system. Besides, a new Attributed Edge Loop Adjacency Graph (AELAG) was introduced and recommended as an alternative method for recognizing 2.5D machining feature. Through this method, important parameters were appended to the recognized 2.5D machining features and this data is useful for generating manufacturing data in AP224 and AP238/ ISO 14649 format and STEP NC data which is important for establishing a more interoperability and intelligent manufacturing environment.

\section{References}

[1] Caligiana, Gianni, Daniela Francia, and Alfredo Liverani. "CAD-CAM integration for 3D hybrid manufacturing." In Advances on mechanics, design engineering and manufacturing, pp. 329-337. Springer, Cham, 2017. https://doi.org/10.1007/978-3-319-45781-9 34

[2] Chlebus, E., and K. Krot. "CAD 3D models decomposition in manufacturing processes." Archives of Civil and Mechanical Engineering 16 (2016): 20-29. https://doi.org/10.1016/j.acme.2015.09.008

[3] Hitomi, Katsundo. Manufacturing systems engineering: a unified approach to manufacturing technology, production management and industrial economics. Routledge, 2017. https://doi.org/10.1201/9780203748145

[4] Hu, Po, Zhenyu Han, Hongya Fu, and Dedong Han. "Architecture and implementation of closed-loop machining system based on open STEP-NC controller." The international journal of advanced manufacturing technology 83 , no. 5-8 (2016): 1361-1375. https://doi.org/10.1007/s00170-015-7631-z

[5] ISO 14649-1. "Industrial Automation Systems and Integration-Physical Device Control-Data Model for Computerized Numerical Controllers-Part 1: Overview and Fundamental Principles." Part 1: Overview and fundamental principles, ISO TC 184/SC1 (2003).

[6] Pandey, Rohit, Arvind Singh Tomar, and Nishant Sharma. "A recent role of CAD/CAM in designing, developing and manufacturing in modern manufacturing technologies." Imperial Journal of Interdisciplinary Research (IJIR) 2, no. 3 (2016): 398-401.

[7] Saha, Samar K., and C. K. Sarkar. "Introduction to technology computer aided design." In Technology Computer Aided Design: Simulation for VLSI MOSFET, p. 1. CRC Press, 2013.

https://doi.org/10.1201/b14860-2

[8] Serksnis, Tony. "Materials and Processes." In Designing Electronic Product Enclosures, pp. 59-111. Springer, Cham, 2019. https://doi.org/10.1007/978-3-319-69395-8 4

[9] Xu, Xun. Integrating advanced computer-aided design, manufacturing, and numerical control: principles and implementations. Hershey, PA: Information Science Reference, 2009. https://doi.org/10.4018/978-1-59904-714-0

[10] Xu, Xun, and Andrew Yeh Ching Nee, eds. Advanced design and manufacturing based on STEP. Springer Science \& Business Media, 2009. https://doi.org/10.1007/978-1-84882-739-4

[11] Zhao, Yaoyao F., Salah Habeeb, and Xun Xu. "Research into integrated design and manufacturing based on STEP." The International Journal of Advanced Manufacturing Technology 44, no. 5-6 (2009): 606. https://doi.org/10.1007/s00170-008-1841-6 